\title{
Development of an Automatic Direction Control System for TBM
}

\author{
Sumio Uematsu*, Tsutomu Nakanishi"*, Shigeru Takamizawa*"*, \\ Kotaro Matsueda ${ }^{* * *}$, Toyoichi Nanbu ${ }^{* \cdots * * *}$, Satoshi Fukuhara ${ }^{* \cdots * * *}$
}

- Civil Engineering Technology Dept. Technology Development Division, Kumagai Gumi CO., LTD.

** Applied Technology Dept. Technology Development Division, Kumagai Gumi CO., LTD.

*** Yokohama Branch, Kumagai gumi CO., LTD.

*..* Construction Plant \& Machinery Dept. Civil Engineering \& Architectural Control Division, Kumagai gumi CO., LTD.

...** Electrical System Design Group. Technical Center, Underground Machinery Division, Komatsu LTD.

***** Research Dept.4. Research Center, Research Division Komatsu LTD.

\begin{abstract}
It is said that the TBM technique will be applied more extensively in Japan. Such prediction refers to these facts : tunnel boring machines applicable to various geological conditions in our country have been developed, and a practice using a TBM is now being studied, for cutting a drift of an extremely large section tunnel. In this context, it is essential to develop an automatic direction control system of TBMs, when considering high-level skill required for operation of TBMs and a great amount of time needed for training of skilled operators.

The system discussed in this paper has been working for a TBM for "construction of the Tsukui headrace tunnel" (TG540-manufactured by Komatsu) since October 1995.
\end{abstract}

\section{Introduction}

About 30 years have elapsed since the TBM technique was introduced into tunnelling sites in Japan. At the first stage, it attracted attention because of its high speed boring capability. Open type TBMs were designed essentially for application to homogeneous hard bedrock. Consequently they did not work very satisfactorily in sites in Japan with a remarkable variety of geological conditions. With the oil crisis in the late 1970s as a momentum, TBMs applicable for any kind of geology were developed for efficiently cutting small- and medium-sized headrace tunnels. On the other hand, use of a TBM is now being studied energetically, for cutting drifts for safe and rapid tunnelling with a section of more than 200 square meters in the second Tomei-Meishin Project.

Under the circumstances, the TBM technique has come to the fore again. Main problems relating to the practice are the following. 
Tunnelling operation is a hard work in a limited space where vibration, dust and mine water are considerable. The operation of TBMs for achieving accurate alignment depends on operator's skill. Training of operators requires a great amount of time.

As a technical solution for these difficulties, an automatic direction control system has been developed and put into practical use, which was applied to a lattice-type thrust jack TBM, one of all-geology type TBMs. The TBM of this type, employed in Japan for the first time, can offer a relatively wide work space. The structure under construction presented in this paper is a headrace tunnel of $5.4 \mathrm{~m}$ boring diameter and $4.690 \mathrm{~m}$ tunnelling distance (for the lot to be executed by the TBM). The direction control system developed has alleviated the hardness of work and facilitated the alignment control.

These are essential items for which technical solutions were necessary in order to develop and put into practical use the direction control system.

1 Antivibration devices are necessary for measuring instruments, since the TBM produces strong vibration (about $10 \mathrm{G}$ ) in the course of boring.

2 Reliability of measurement systems must be ensured in the dusty environment with mine water.

3 Variation in posture and trajectory of the lattice-type thrust jack TBM should be analyzed precisely.

\section{Summary of the lattice-type thrust jack TBM}

\subsection{Summary of the TBM}

The TBM (TG540), to which the direction control system is applied, is composed of a front-shield and a rear-shield. The front-shield is provided with cutter, front-side jacks. The rear-shield is equipped with main grippers, shield jacks. Between the front-shield and rear-shield, 12 thrust jacks are installed. This machine has a shield structure with cylindrical skin plates to cope with adverse ground conditions.

The front-side jacks help maintaining the posture of the front-shield when pulling forward the rear-shield and during excavation. The main grippers secure the rear-shield against the tunnel wall and receive the reaction of the advancing front-shield. The shield jacks produce reaction from invert segments, supplementing thereby the advancing force when the reaction from the main grippers is insufficient due to adverse geology. The thrust jacks are arranged with an inclination on the shield, named "lattice-type thrust jack" system. Such design propels the machine, controls the direction and receives the torque reaction due to cutter rotation during excavation.

Photo 1 shows the main structure of the TBM, Fig.1 the configuration of the machine, and Table 1 the machine specifications. 


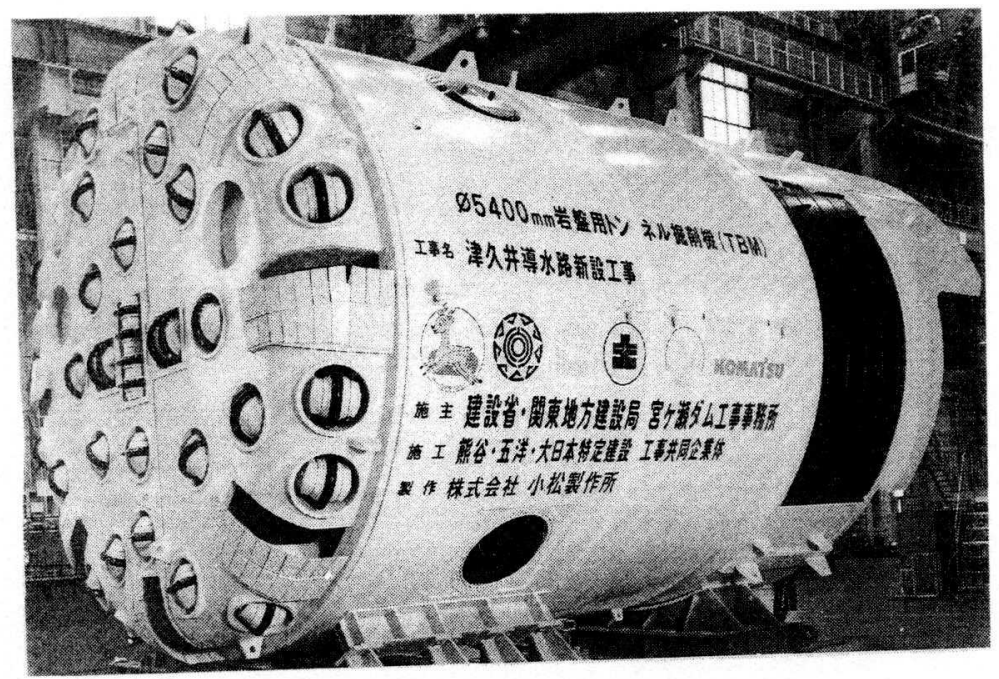

Photo 1 TG540 (made by Komatsu)

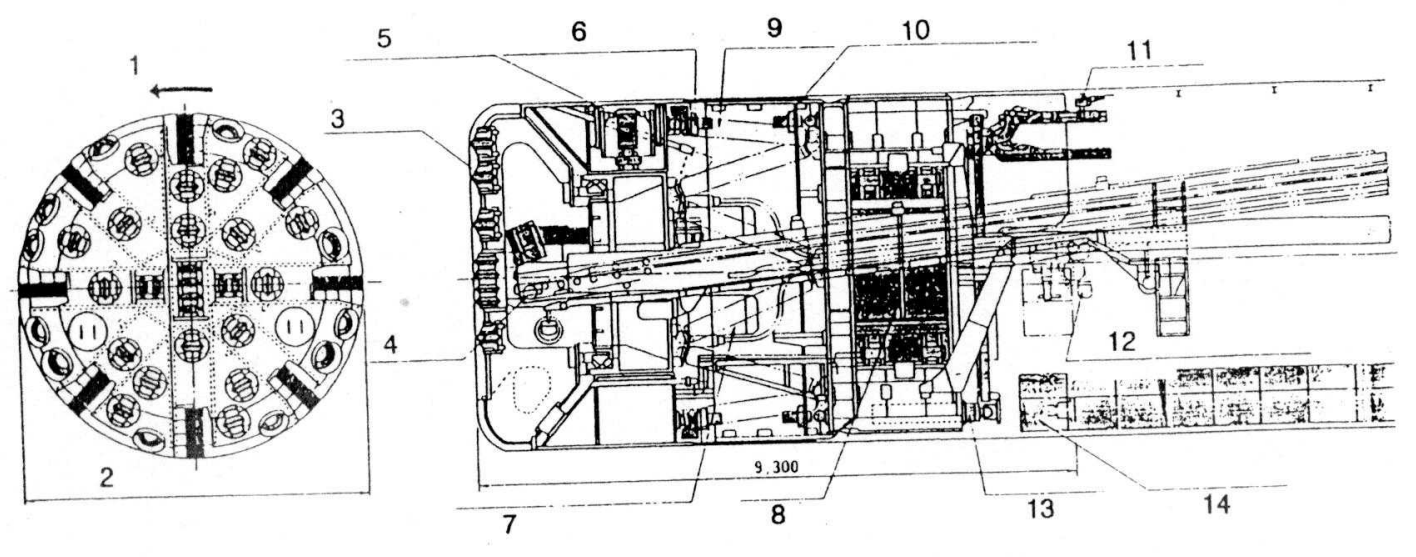

Legend
1. Rotation direction
6.Articulated jack
11. Support erector
(Lifting capacity 0.5 t)
2. Boring diam. $5,400 \mathrm{~mm}$
7. Geared-motor for driving
12. Invert segment conveyor/ installer
(Lifting capacity 3.5 t)
3. Disc cutter
8. Gripper
13. Shield jack
4. Belt conveyor
9. Thrust jack (lattice type)
14. Invert segment
5. Front side jack
10.Jack for adjusting sliding portion

Fig.1 Configuration of TG540 
Table 1 Specification of the TBM (TG540)

\begin{tabular}{|l|l|}
\hline Boring diameter & $5,400 \mathrm{~mm}$ \\
\hline Machine length & $9,300 \mathrm{~mm}$ \\
\hline Weight & $\begin{array}{l}\text { Shield and Main equipment: } 240 \mathrm{t} \\
\text { Rear wagon: } 110 \mathrm{t}\end{array}$ \\
\hline Total thrust & about $1,400 \mathrm{kw}$ \\
\hline Disk cutter & 17 inches $(432 \mathrm{~mm}$ ) diam. $\times 37$ units \\
\hline $\begin{array}{l}\text { Rotation speed, torque } \\
\text { of cutter }\end{array}$ & $6.3 \mathrm{rpm}, 139 \mathrm{t}-\mathrm{m}$ \\
\hline Thrust jacks & $155 \mathrm{tf} \times 1,600 \mathrm{~mm}$ stroke $\times 6$ sets $(12$ jacks) \\
\hline Grippers & $1,000 \mathrm{tf}$ for each side (extension $200 \mathrm{~mm}$ each) \\
\hline Front side jacks & $100 \mathrm{tf} \times 135 \mathrm{~mm}$ stroke $\times 4$ jacks \\
\hline Shield jacks & $150 \mathrm{tf} \times 1,150 \mathrm{~mm}$ stroke $\times 2$ jacks \\
\hline
\end{tabular}

\subsection{Driving motion of the TBM}

A cycle of driving motion is consisted of : securing the rear-shield (extending the main grippers), driving (steering or advancing), securing the front-shield (extending the frontside jacks), preparation for the next cycle (pulling forward the rear-shield). Fig.2 illustrates the structure of lattice-type thrust jacks and Fig. 3 the sequence of a driving cycle.

The driving force of the machine is given by the 12 lattice-type thrust jacks. The jacks work in two modes, steering and advancing. The machine goes forward by repeating the two modes. In the steering mode, the location and direction of the front-shield is corrected, then in the advancing mode the machine is forwarded straight.

The hydraulic system for the thrust jacks include 2 sets of 6-grouped pumps, which feed each jack separately with a same amount of oil, extending thereby the jacks at a same speed. The machine advances, receiving the forwarding thrust and torque at the same time (advancing mode).

When controlling the direction of the machine, several jacks in the opposite side of the desired direction are fed with oil to make a difference in jack stroke (steering mode), which produces an angle of the front-shield with respect to the rear-shield, then all the jacks are fed with a same amount of oil by the independent pumps. This function enables the machine to advance under the thrust of all the jacks, while maintaining the angle of the front-shield.

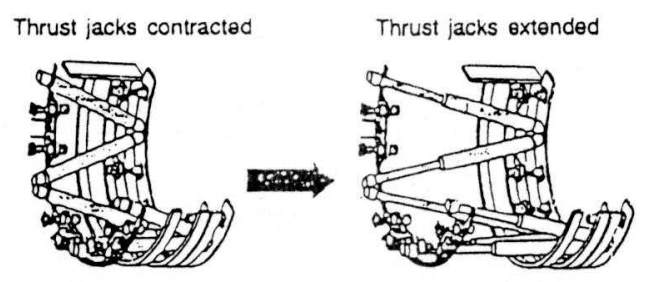

Fig. 2 Lattice-type thrust jacks 

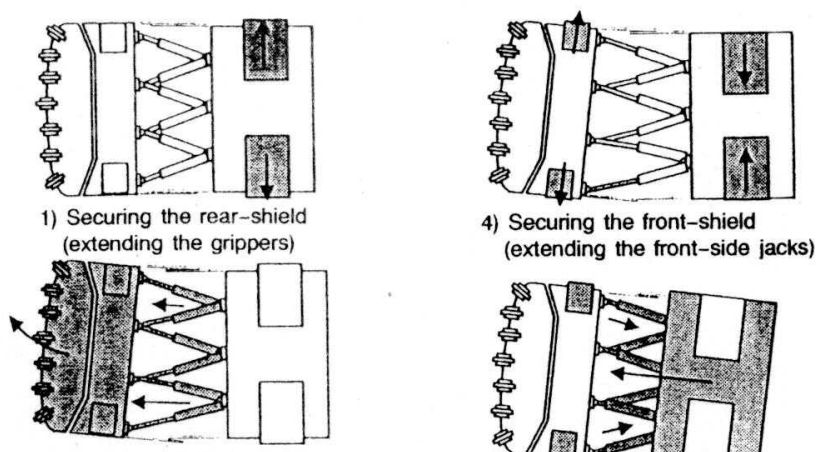

2) Driving (steering)
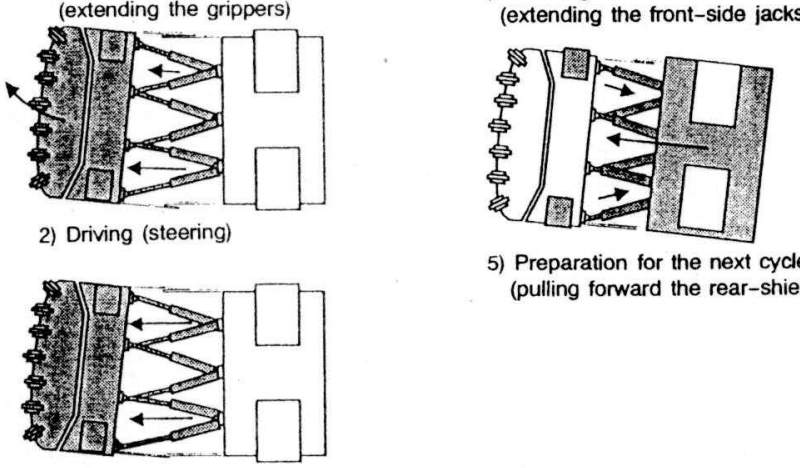

5) Preparation for the next cycle (pulling forward the rear-shield)

3) Driving (advancing)

Fig.3 Driving motion

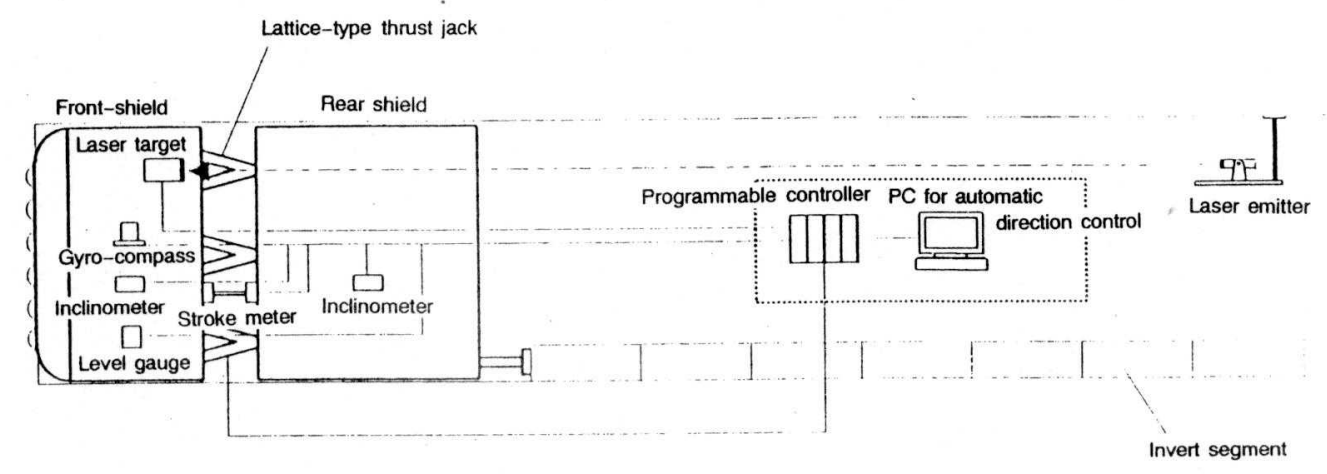

Fig. 4 Configuration of the system

\section{Automatic direction control system}

\subsection{Configuration of the system}

Fig. 4 is a schematic diagram of the system configuration.

The system is composed of : laser emitter distant from the rear-shield; laser target, gyro-compass, level gauge, inclinometer in the front shield; inclinometer in the rear shield; stroke-gauges for measuring the extension of the thrust jacks between front- and rear-shields. Besides, each jack is provided with a stroke-gauge and a pressure gauge. The data from the measurement instruments are utilized in analysis for creating an algorithm and in monitoring troubles of the machine such as sliding of the rear-shield while driving.

The data outputted from the sensors are analyzed and processed in real time by the personal computer installed in the operation room for direction control . 
The personal computer collects and stores in real time the data given by the laser system, and analyzes them to display in real time on the monitor the current driving situation of the machine. Besides such function, in the advancing mode the computer continuously detects vertical and horizontal deviations of the front-shield from the driving target line. When the machine position exceeds the target range (insensitive range), the mode is shifted to "steering" automatically, calculates and sends to the programmable controller the direction control amount for returning the front-shield to the driving target line. The calculation of the control amount is performed by processing the current position data of the front-shield, posture data of the front-shield and that of the rear shield. The processing is based on the automatic direction control algorithm established through analysis of foregoing driving data.

\subsection{Control technique}

For establishing the automatic direction control algorithm, data of the TBM behavior have been collected during manual operation.

Analysis of the collected data demonstrated the following characteristics of the machine behavior.

$1^{\circ}$ When steering operation is done, the position and posture of the front-shield are changed in the steering direction. If driving is done without steering operation (advancing mode), the front-shield goes in the orientation of the rear-shield.

$2^{\circ}$ If the posture of the front-shield is excessively changed by steering, the orientation of the rear-shield varies in the direction opposite to the steering direction.

Taking into full account the machine behavior above, a scheme of direction control has been established; i.e.,

$1^{\circ}$ A target line for advance of the machine is set up. During driving, direction control is done so that the front-shield is located on the target line.

$2^{\circ}$ Some amplitude (range) is given to the target line as shown in Fig. 5. When the front-shield exceeds the range, steering operation is performed for repositioning the front-shield in the target range.

$3^{\circ}$ A deviation limit of the posture of the front-shell from the target direction is set up for direction control.

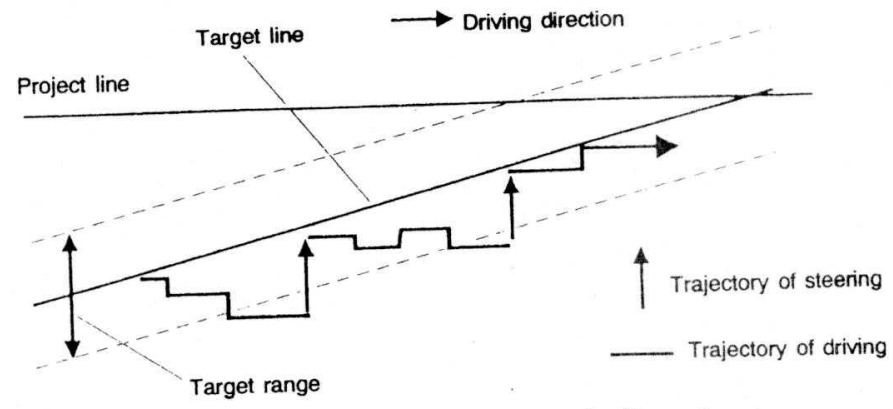

Fig. 5 Principle of control direction 
Fig. 6 shows the flow of the automatic direction control.

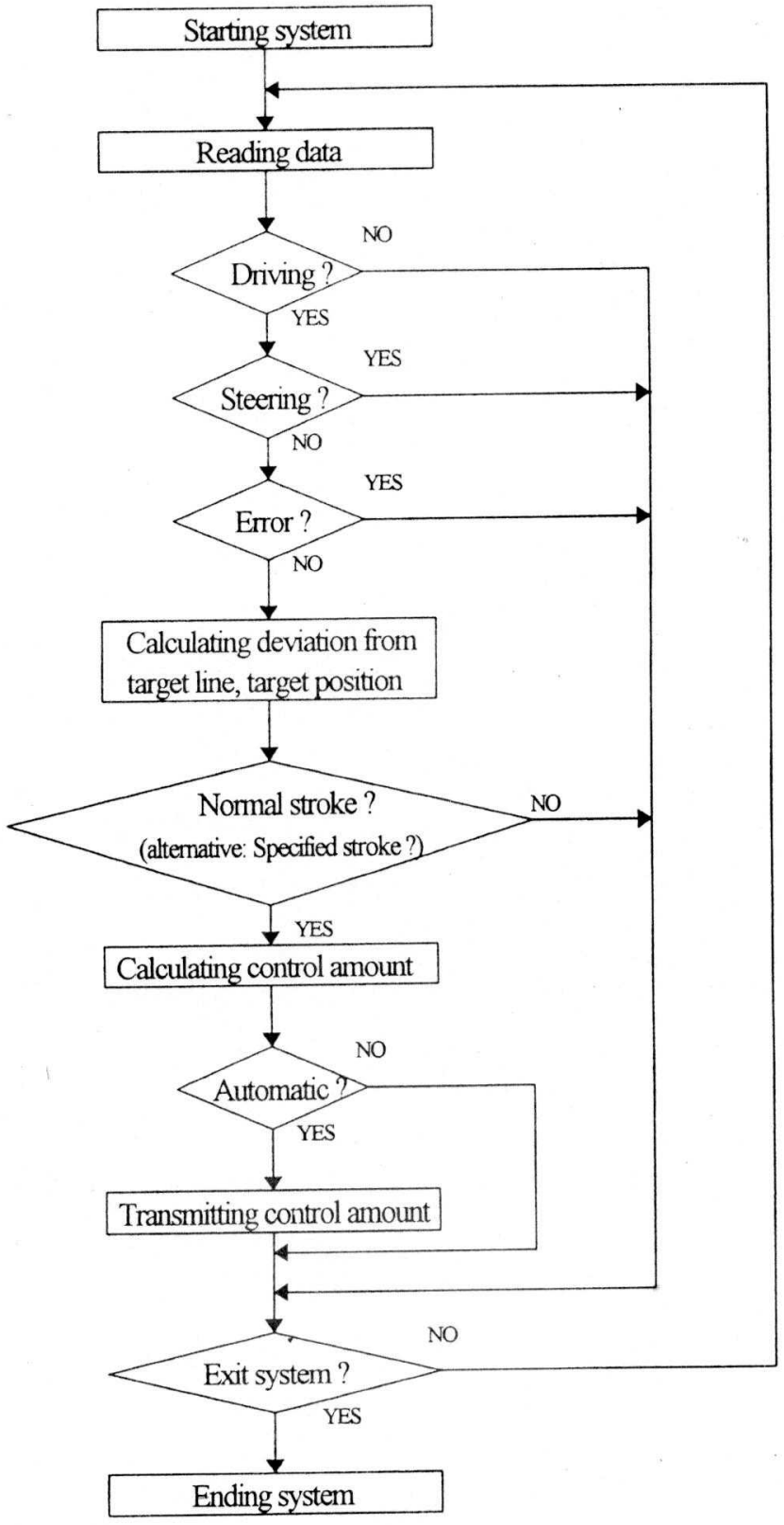

Fig.6 Automatic control direction flow

The main steps of the flow and functions of the control system are explained in the following.

(1) Error check

When a problem concerning the direction control occurs, which needs operator's judgment "whether or not the automatic direction control should be continued", an indication of "error" appears on the monitor to emit an alarm from the control panel for the operator. In this system "machine position data" are obtained by laser measurements. 
When a person prevents the laser path for a considerable span of time, the position data essential for direction control are not renewed. In such a case, an "error" indication is given to command the operator to free the laser path.

(2) Setting up the target line and calculating the deviation from the target position

A target line is set up at the time of beginning of one driving cycle. The line is determined by the target position at the beginning of one cycle, final target position and target approaching distance (see Fig. 7).

By determining a target line for each cycle, the target line approaches the project line gradually as the machine position reaches the target position. The frequency of steering operation is accordingly reduced gradually.

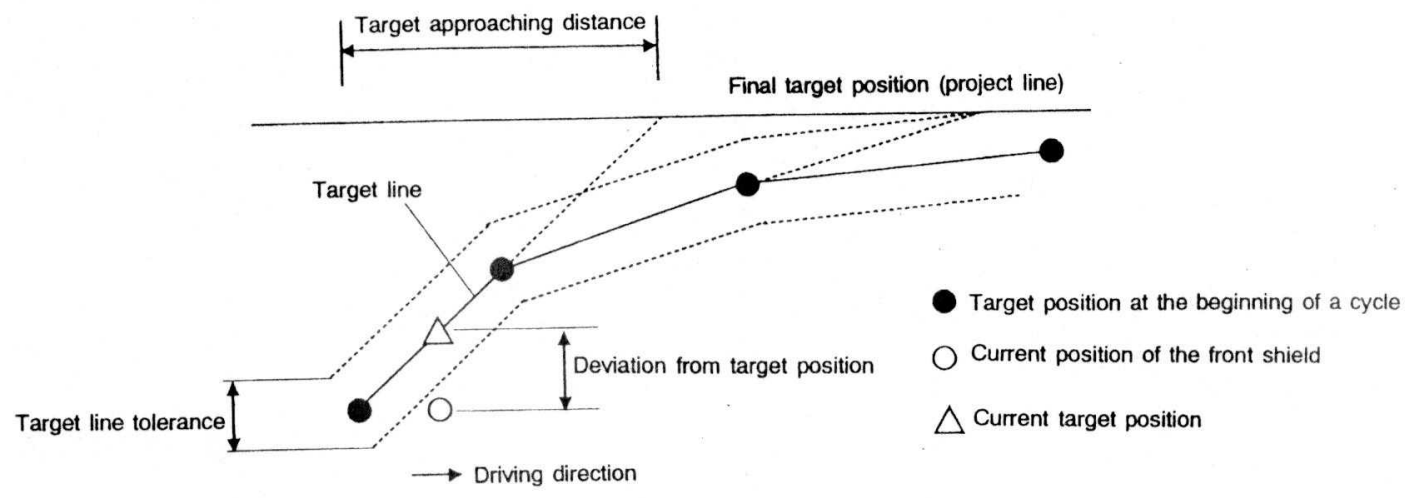

Fig. 7 Target position, deviation from target position and target approaching distance

(3) Calculating the control amount

The control amount is calculated when the front-shield exceeds the target range.

On the basis of the horizontal and vertical deviations from the target position and the number of repetitive operations in the prevailing direction steering steps are determined.

The prevailing direction means the direction, horizontal or vertical, which has priority in direction control.

For example, the prevailing direction is vertical and vertical steering cycle has been repeated more than three times. If, after steering cycle repeated more than three times, the vertical target is not reached sufficiently, horizontal steering is performed for smooth direction control.

The control amount outputted in such operation represents the stroke difference (interval difference between front- and rear-shields in the horizontal or vertical direction) to be achieved by automatic steering.

The control amount is calculated from two parameters, "deviation amount from the target position" and "deviation angle from the reference posture of the front-shield".

The "stroke difference" is obtained from the relationship between "stroke difference" and "correction amount of position", which has been determined by statistic processing, and from the "deviation amount from the target position".

The actual control amount (stroke difference) is given so that the orientation of the rearshield should not be changed under the influence of steering action, referring to the current posture of the rear-shield, as follows. 
1 Since the posture of the front-shield changes when a "stroke difference" is produced, the "stroke difference" is reduced if the deviation angle of the front-shield from the reference posture is expected to increase.

2 In order to avoid an excessively large change of the posture of the front-shield, the "stroke difference" output is reduced if the "stroke difference" once calculated for controlling is excessively large.

\section{Application to the practical work}

The control system has been working for direction control, while collecting operation data, since it was mounted in October 1995 on TG540 driving the Tsukui headrace tunnel.

\subsection{Summary of the tunnelling work}

Name of the work: Construction of new Tsukui headrace

Location: from Aikawa-cho, Aiko-gun to Tsukui-cho, Tsukui-gun, Kanagawa Prefecture

Period: $\quad$ from February 1993 to March 1996

Owner: $\quad$ Ministry of Construction, Kanto Regional Construction Bureau, Miyagase Dam Construction Office

Contractors: Joint venture of Kumagai, Goyo, Dainippon for special construction work Tunnel length: 5,159.9 $\mathrm{m}$ (including the lot driven by TBM of 4,690 m)

Inclination: 1/1000 (downward)

Alignment: with a curve (300 $\mathrm{m}$ in curvature radius)

Tsukui headrace was projected, along with Doshi headrace, to efficiently link and operate Miyagase dam, Sagami dam and Shiroyama dam to be constructed in Sagami-gawa river line, achieving thereby optimum utilization of the precious water source.

The tunnelling of Tsukui is performed by the TBM over a length of about 4,700 $\mathrm{m}$ on the inlet side (the tunnel is $5,159 \mathrm{~m}$ long in total).

The tunnel boring machine was assembled in the starting base on the inlet side, then began to advance toward the outlet. Before collection of data on the machine behavior, some engineering solutions have been taken, that is, use of antivibration rubber, high performance dust collector and higher power laser emitter.

\subsection{Results of the application}

Good results were obtained by using the direction control system (See Tables 2, 3, Figs. $8,9)$.

In the case of the TBM discussed here, steering operation decreases the driving speed, that is, unnecessary steering operations elongate the driving time.

Through comparison between operation records of "automatic" and "manual" modes, it has been shown that the frequency of steering is slightly higher in the "automatic mode". As for the total driving time, the results are almost the same for both modes.

It was because steering operations were done more minutely in the "automatic mode", but there was no significant difference in the total operation time. 
Table-2 Work conditions for the present case

\begin{tabular}{|l|l|l|}
\hline & \multicolumn{1}{|c|}{ Position control } & \multicolumn{1}{c|}{ Posture control } \\
\hline Vertical & $\begin{array}{l}\text { Shifts the position deviation } \\
\text { upward by } 5 \text { to } 20 \mathrm{~mm} . \\
\text { (Target value varies with } \\
\text { driving distance.) }\end{array}$ & $\begin{array}{l}\text { Admissible if the posture is } \\
\text { within the tolerance range } \\
\text { around the reference value. } \\
\text { (Vertical, Horizontal directions) }\end{array}$ \\
\hline Horizontal & $\begin{array}{l}\text { The current state is maintained } \\
\text { with no shift (0mm). }\end{array}$ & \\
\hline
\end{tabular}

Table-3 Application results (comparison with target and reference values)

\begin{tabular}{|l|c|c|}
\hline & Position control accuracy & Posture control accuracy \\
\hline Vertical & -10 to $15 \mathrm{~mm}$ & -15 to $15 \mathrm{~min}$. \\
\hline Horizontal & -15 to $15 \mathrm{~mm}$ & -0.3 to $0.2 \mathrm{deg}$. \\
\hline
\end{tabular}

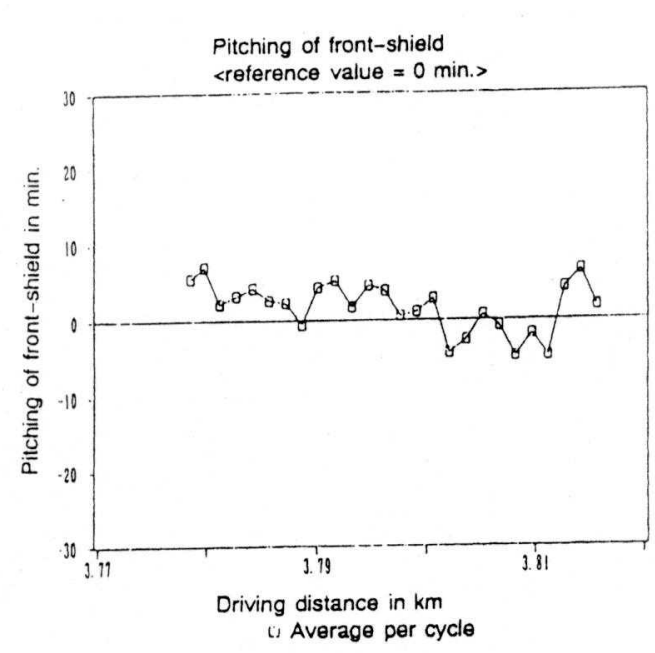

Fig. 8 Results of posture control

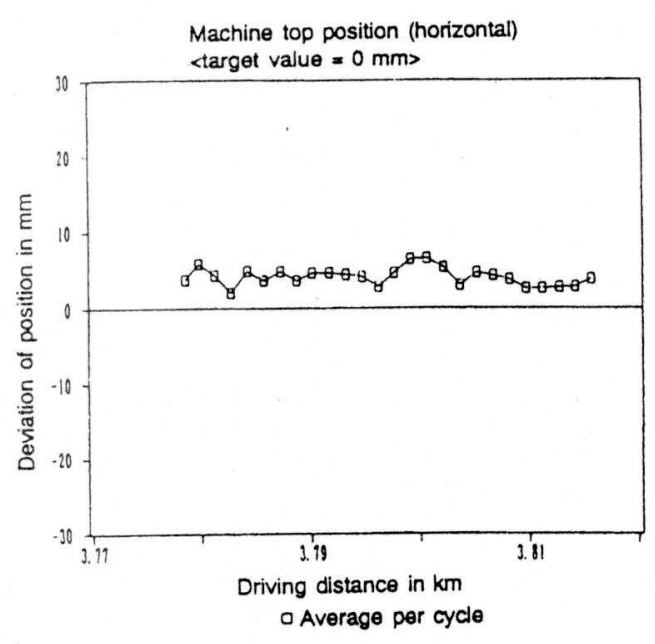

Fig. 9 Results of position control

\section{For further development}

Data collection of practical operations will be continued in another site for further enhancing the applicability of the system. Considering the trend toward more extensive use of tunnel boring machines in the Second Tomei-Meishin Highway Project for example, the author et al. are intending to establish an integrated management system of work by TBM on the basis of the records of the automatic direction control system. 